\title{
Publisher's Note: Fractional topological phases in three-dimensional coupled-wire systems [Phys. Rev. B 92, 115152 (2015)]
}

\author{
Tobias Meng \\ (Received 1 February 2016; published 11 February 2016)
}

DOI: 10.1103/PhysRevB.93.079901

This paper was published online on 28 September 2015 with an error in Ref. [59]. Reference [59] should read as " $Z$. Wang, Physica B 475, 80 (2015)." The reference has been corrected as of 27 January 2016. The reference is incorrect in the printed version of the journal. 